\title{
But I told you she was ill! The role of families in preventing avoidable harm in children
}

\section{Damian Roland ${ }^{1,2}$}

${ }^{1}$ SAPPHIRE Group, Health Sciences, Leicester University, Leicester, UK

${ }^{2}$ Paediatric Emergency Medicine Leicester Academic (PEMLA) Group, Leicester Royal Infirmary, Leicester, UK

\section{Correspondence to}

Dr Damian Roland, SAPPHIRE Group, Health Sciences, Leicester University, Leicester LE1 6TP, UK; dr98@le.ac.uk

Accepted 9 January 2015 Published Online First

27 January 2015

\section{SLinked}

- http://dx.doi.org/10.1136/ bmjqs-2014-003001

\section{CrossMark}

To cite: Roland D. BMJ Qual Saf 2015;24:186-187.
"But I told you they were ill!" These are chilling words for any healthcare professional to hear if harm has occurred to a child following failure to recognise a serious illness. Failing to detect and act on a child who is deteriorating is an important form of avoidable harm that remains a significant issue both in and out of hospitals. ${ }^{12}$

Systems for tracking illness in children and triggering clinical response have been in use for some time, with a variety of Paediatric Early Warning Systems (PEWS) available. ${ }^{3}$ Typically, these systems use features common to clinical observations such as heart rate, respiratory rate and temperature. But something that draws on the skills and knowledge of the person who knows the patient best-usually the parents-could be a very welcome addition to improving recognition of the deteriorating child. Yet parental concern is only used in a minority of PEWS systems ${ }^{4}$ in the UK (with some evidence of greater use in the USA). ${ }^{5}$ One possible reason for low rates of parental involvement in monitoring of their child's condition in hospital is concern about how it might be optimally deployed, and such concern may be amplified when it is proposed to give parents a direct role in escalating clinical response.

In their work, Brady et $a l^{6}$ examined the use of family activation of a medical emergency team (MET) in a large tertiary children's hospital. They note that potential concerns associated with families' involvement in activating these teams might include the risks that the system might be abused, divert interventions away from those in need or require additional resources for training that are not available. That these concerns are paternalistic is almost ironic, so it is pleasing to see an organisation attempt to investigate them and involve parents and carers in every stage of the improvement initiative. Brady et al's ${ }^{6}$ study demonstrated that family-activated MET calls were only a small proportion of the total MET calls made. This proportion was also far less than falsepositive clinician-led calls (ie, an unnecessary activation with no benefit for the patient), suggesting that families were not stressing the system unnecessarily.

What does this mean for hospitals currently using a PEWS-type or MET-type system that are considering the introduction of a family-activated component? For one, they should not expect to be deluged with MET alerts. However, as the authors recognise, much effort may need to be invested in informing families about the process. The authors admit the hospital failed to collaborate with parents initially and required a revision of their information posters as a result. This highlights the importance of coproduction and is an important lesson for hospitals.

A second issue relates to the context in which the intervention was developed and delivered: one that already had an excellent safety culture, including, for example, patient advocates at all team huddles. Deployment of a family-activated MET calls may depend on leadership that allows for honest and critical reflection on its own communication processes. The very fact the authors of the Cincinnati study identify family-led MET calls that did not result in a paediatric intensive care unit transfer as ".... positive communication threat"-rather than a false positive, for example-implies a cultural attitude in that organisation that supports family engagement. Patient-centred leadership was also demonstrated by the brave decision to allow families to call METs directly via their hospital's switchboard since a less courageous organisation might have worried about a communication dynamic that seemed to suggest a family no longer 
trusted their allocated nurse or doctor. Whether delivery and outcomes would be similar in a hospital still developing a robust patient safety programme and cultural disposition is an open question.

For organisations willing to implement familyactivated MET calls, a great deal can be learned about quality improvement from this paper. These include the recognition that a number of improvement initiatives were ongoing during the study, and that some may well have had an impact on the intervention. This should not detract from the results, as rarely does one intervention alone that transforms a safety culture. Second, learning from the improvement initiative itself was valuable in its own right:

We also have observed at our centre that the "threat" of a family-calling an MET can empower a nurse to do so even when the physician team disagrees with the MET's necessity.

While the language of 'threat' may not be a culture an organisation wishes to endorse, the cascade of delivering impact from one mechanism (the family-activated MET call) to another (the empowerment of individual staff) is clear. One improvement approach may contribute to another. Recognising and sharing this is beneficial for understanding the effectiveness of the intervention.
With coproduction and strong leadership, it may be possible to consign those heart-breaking words "I told you they were ill" to the past.

Twitter Follow Damian Roland at @damian_roland Competing interests None.

Provenance and peer review Not commissioned; internally peer reviewed.

\section{REFERENCES}

1 Wolfe I, Macfarlane A, Donkin A, et al. Why children die: death in infants, children, and young people in the UK-Part A. Royal College of Paediatrics and Child Health, 2014.

2 Mellor J. An avoidable death from a three-year-old child with sepsis. Health Service Ombundsman, 2014.

3 Roland D. Early warning scores: Holy Grail and Achilles Heel. Arch Dis Child Educ Pract Ed 2012;97:208-15.

4 Roland D, Oliver A, Edwards E, et al. Use of paediatric early warning systems in Great Britain: has there been a change of practice in the last 7 years? Arch Dis Child 2014;99:26-9.

5 Sen AI, Morgan RW, Morris MC. Variability in the implementation of rapid response teams at academic American pediatric hospitals. J Pediatr 2013;163:1772-4.

6 Brady PW, Zix J, Brilli R, et al. Developing and evaluating the success of a family activated medical emergency team: a quality improvement report. BMJ Qual Saf 2015;24: 203-11. 